\title{
DISCONTINUOUS SOLUTIONS IN THE CALCULUS OF VARIATIONS*
}

\author{
BY L. M. GRAVES
}

1. Introduction. By a discontinuous solution in a calculus of variations problem is meant an extremizing arc having one or more corners, that is, the derivatives of the functions defining the arc have one or more ordinary discontinuities. Such discontinuities may occur when the extremizing curve is required to have a point in common with the boundary of the region where admissible curves must lie, or when the integrand function is discontinuous. These and other cases will be discussed later. But I wish to take up first the case when the function to be minimized (or maximized) is a simple integral of the form

$$
J=\int_{t_{1}}^{t_{2}} F\left(x, y, x^{\prime}, y^{\prime}\right) d t,
$$

where the integrand $F$ has the usual continuity properties, and the minimizing curve is wholly interior to the region of admissible curves. When the problem is not regular, corners are very likely to occur on the minimizing arc, even when the two endpoints can be joined by an extremal without corners. This depends on the behavior of the Weierstrass $\varepsilon$-function, as will be seen later. Examples are $F=\left(a x^{\prime 2}+2 b x^{\prime} y^{\prime}+c y^{\prime 2}\right)^{1 / 2}$, where the quadratic form is positive definite and the coefficients $a, b, c$ are properly chosen functions of $x, y, x^{\prime}, y^{\prime}$; and, for a non-parametric problem, $f=y^{\prime 2}\left(y^{\prime 2}-\phi(x, y)\right)$, where $\phi$ is positive.

2. The Minimizing Curve is Interior to the Region of Admissible Curves. The first additional necessary conditions on a minimizing arc with corners are the Weierstrass-Erdmann corner conditions, which state that $F_{x^{\prime}}$ and $F_{y^{\prime}}$ must be continuous along that arc. These conditions were given by Weierstrass in 1865. $\dagger$ For the non-parametric problem, the two functions

* An address presented at the invitation at the program committee at the meeting of the Society in Ann Arbor, November 29, 1929.

$\dagger(51)$, vol. 7 , pp. 109, 250. The numbers in parentheses in foot notes refer throughout to the bibliographical list at the end. 
$F_{x^{\prime}}, F_{y^{\prime}}$ are to be replaced by $f-y^{\prime} f_{y^{\prime}}, f_{y^{\prime}}$, and the conditions were given by Erdmann in 1876.* Todhunter obtained the continuity of $f_{y^{\prime}}$ in the special case when $f$ depends only on $y^{\prime}$, in $1871 . \dagger$

Nothing more of note was done about discontinuous solutions in the case we are considering until the dissertation of Carathéodory in 1904. $\ddagger$ It seems to have been the fashion to restrict attention to regular problems, in which corners cannot occur. Carathéodory pointed out the unwisdom of excluding from consideration such a large class as the non-regular problems. He also proved a theorem which shows why corners are likely to occur on a minimizing arc. Consider an extremal arc $E$ along which

$$
\mathcal{E}(x, y, \cos \theta, \sin \theta, \cos \bar{\theta}, \sin \bar{\theta})>0
$$

whenever the direction $\bar{\theta}$ is different from the direction $\theta$ of the extremal. Suppose we extend $E$ until a point $P_{0}$ is reached at which this property fails to hold. Then, in general, a corner is possible at $P_{0}$, and the second extremal arc in the new direction will also satisfy the condition (1) of Weierstrass except at the initial point $P_{0}$. Furthermore, if we proceed past the point $P_{0}$ on the original extremal $E$, there will be directions $\bar{\theta}$ for which the Weierstrass function $\varepsilon$ is negative.

The extension of the Jacobi condition to discontinuous solutions is somewhat complicated. I shall outline the principal facts in the non-singular plane case, $\S$ and then point out briefly the contributions of Carathéodory, Bolza, Dresden, and Graves to the theory.

Consider a minimizing arc having a single corner, and hence composed of two arcs of extremals, $E^{-}$and $E^{+}$. If $\phi^{-}$is a oneparameter family of extremals containing $E^{-}$, then in general a complementary family $\phi^{+}$containing $E^{+}$and a corner curve $C$ are determined, such that extremal arcs of the two families may be pieced together to form broken extremals or extremaloids, with corners on $C$ and satisfying the corner conditions. The slope of the corner curve $C$ at the point $P_{0}$ where $E^{-}$joins

* (1).

$\dagger(48)$, pp. 2,3 .

$\ddagger(2)$.

§ See Bolza (54), pp. 372-388. 
$E^{+}$, the focal point $Q^{-}$of the family $\phi^{-}$on $E^{-}$, and the focal point $Q^{+}$of the family $\phi^{+}$on $E^{+}$are intimately related. In fact when one of the three is given the other two are uniquely determined. Moreover, as the point $Q^{-}$moves toward $P_{0}$ from the point $P_{0}^{-}$conjugate to $P_{0}$ on $E^{-}$, the tangent to the corner curve rotates always in one direction through $180^{\circ}$, starting from a position of tangency to $E^{-}$. The position of $Q^{-}$corresponding to tangency of the corner curve to $E^{+}$we denote by $T^{-}$. At the same time the point $Q^{+}$moves from an initial position $T^{+}$out toward the point $P_{0}+$ conjugate to $P_{0}$ on $E^{+}$, and then as $Q^{-}$moves from $T^{-}$to $P_{0}, Q^{+}$moves from $P_{0}$ to $T^{+}$. Necessary conditions for a minimum are: (1) that the first endpoint, $P_{1}=Q^{-}$, shall follow $T^{-}$, and (2) that the second end-point $P_{2}$ shall precede the point $Q^{+}$conjugate (in the nerw sense) to $Q^{-}$.

Carathéodory* in his dissertation showed how to construct families of extremaloids, $\dagger$ discovered the points we have denoted by $T^{-}$and $T^{+}$, and knew the relative motion of the two focal points $Q^{-}$and $Q^{+}$and the tangent to the corner curve. Then he proved the second of the necessary conditions mentioned above quite simply by means of the extension of the Kneser envelope theorem. He seemed to realize also (though he gave no proof) that the corner curve must cross the extremaloid at the corner. Now this in view of the other conditions is equivalent to the first condition, as Dresden's work showed. Bolza clarified some details of the situation and gave an analytic proof regarding the motion of the points $Q^{-}$and $Q^{+}$and the tangent to the corner curve. Finally in the same year Dresden $\S$ showed that the minimizing property of an arc with a corner stops at the points $T^{-}$and $T^{+}$.

Consider now the one-parameter family of extremals passing through the point $P_{1}$, and the complementary family which contains the arc $E^{+}$. These two families make up a one-parameter family of extremaloids whose equations may be written $x=x(t, a), y=y(t, a)$. If $D(t)$ denotes the determinant $x_{t} y_{a}-y_{t} x_{a}$ evaluated along the extremaloid $E$, then the condition (1) given above is equivalent to (1a) $D(t)$ does not vanish nor change sign

*(2).

$\uparrow$ See Rider (10), Sakellariou (11), (12).

$\ddagger(4)$.

$\S(5)$, pp. $480 \mathrm{ff}$. 
at the corner, while (2) is equivalent to $(2 a) D(t)$ does not vanish between the ends of the minimizing arc. In my recent paper* I have proved the two conditions in this form simultaneously by means of the second variation. Space forbids going into details here. Suffice it to say that it seems to be necessary to cast the problem in parametric form in order to get an effective extension of the Jacobi condition by means of the second variation. I have also given a direct proof for the condition (1a) by a differentiation method. Both of my methods have the advantage of extending unchanged to the problem in $n$ dimensions. Moreover, several corners cause no additional complication in the statement of the conditions (1a) and (2a). For the Lagrange problem analogous conditions have been derived by means of the second variation in a partially completed dissertation by Hefner. $\dagger$

Both Bolza $\ddagger$ and Graves have given sufficient conditions for a minimum. The latter has shown in this connection that if the Weierstrass function $\varepsilon$ is positive along an extremaloid, then in general it remains positive on neighboring extremaloids of a family. $\S$

In 1923 Razmadzé obtained Dresden's condition relative to the points $T^{-}$and $T^{+}$of Carathéodory by a different method. He gives the following geometric interpretation of the point $T^{-}$. Produce the second arc $E^{+}$of the extremaloid $P_{1} P_{0} P_{2}$ backward to $S$. Construct a family of extremals containing the first arc $E^{-}$, such that each extremal of the family cuts the $\operatorname{arc} S P_{0} P_{2}$ at an angle reducing the Weierstrass function $\varepsilon$ to zero. Then the focal point of this family on the arc $E^{-}$is the point $T^{-}$of Carathéodory. On the basis of this construction, Razmadzé gives a direct proof of the condition that the first end-point $P_{1}$ must not precede the point $T^{-}$.

There is a function $\Omega=x^{\prime}-F_{x}{ }^{+}+y^{\prime}-F_{y}{ }^{+}-x^{\prime+} F_{x}{ }^{-}-y^{\prime}+F_{y}{ }^{-}$ which Dresden $\|$ proved to have an intimate relation with the Weierstrass function $\varepsilon$, and which is a factor of the functional

$*(8)$.

$\dagger(9)$.

$\ddagger(54)$, pp. $381 \mathrm{ff}$.

\& Graves (8), p. 11, Lemma 5.6

I (7).

\| See (5), p. 485 . 
determinant involved in securing the family of extremals $\phi^{+}$ complementary to a given family $\phi^{-}$. A considerable part of the foregoing discussion depends on the assumption that $\Omega \neq 0$ at the corners. In 1913 Carathéodory* treated the relation between $\varepsilon$ and $\Omega$ for the case when $\Omega$ vanishes. On the basis of this paper he promised further developments, which I have not seen in print. The case when $\Omega$ vanishes identically was discussed in the dissertation. $\dagger$ The location of the corners is then undetermined, and extremaloids joining the two endpoints may be put together in infinitely many ways. Dresden obtained the necessary conditions (1) and (2) even in case $\Omega=0$, except that (1) is proved only in the weaker form: $P_{1}$ does not precede $T^{-}$.

Carathéodory also treated isoperimetric problems in his dissertation, paying special attention to the case when both integrals have the same extremals without their Euler equations being identical. His results are not complete. He lays emphasis on this case as being one in which corners must occur.

In his 1906 paper $\ddagger$ Carathéodory considers the Minimum im Kleinen for positive definite but non-regular problems. Consider a bounded closed region in which the integrand $F$ is everywhere positive, and from each point of which there issues a family of extremaloids or broken extremals satisfying the condition (1) except at the corners. Then there is a constant $\rho$ such that the circle with center at a point $P$ and radius $\rho$ is simply covered by the family of extremaloids issuing from $P$. Carathéodory states that with this as a basis, Hilbert's existence proof for the absolute minimum can be carried through under less restrictive hypotheses, but the details have not to my knowledge appeared in print.

In the discussion so far, it has been supposed that the derivatives of the functions defining the minimizing curve had at most a finite number of ordinary discontinuities. The case when there are infinitely many discontinuities has not been satisfactorily treated. In 1901 Whittemore $\S$ obtained the du Bois-Reymond equations

* (6)

$\dagger$ (2). See also Bolza (54), p. 388.

$\ddagger(3)$.

§ (13). 


$$
f_{y^{\prime}}=\int_{x_{1}}^{x} f_{y} d x+c,
$$

for the non-parametric integral, assuming that the set of discontinuities of the derivative $y^{\prime}$ has content zero, and that $y^{\prime}$ is bounded. Hahn treated the parametric integral with analytic integrand in 1907.* Assuming the minimizing curve to be rectifiable and to have a well defined tangent at each point, he showed that it must satisfy the du Bois-Reymond equations almost everywhere. Moreover, the minimizing curve is made up of denumerably many analytic curves satisfying the Euler equations. Under the weaker hypothesis that the minimizing curve is rectifiable and has a forward tangent at each point, Hahn again obtained the du Bois-Reymond equations, and showed that $F_{x^{\prime}}$ and $F_{y^{\prime}}$ are continuous at isolated discontinuities of the tangent direction.

In my dissertation $\dagger$ I treated the non-parametric integral, assuming $y$ to have bounded difference quotients. I obtained the du Bois-Reymond equation almost everywhere, and the Weierstrass and Legendre conditions at points where $y^{\prime}$ is right hand or left hand continuous. Later I showed the existence of a family of solutions of the du Bois-Reymond equation, and obtained a corresponding extension of the Jacobi condition. However, this extension is of little significance, since the family of solutions of the du Bois-Reymond equations has the discontinuities of $y^{\prime}$ on vertical lines, and the condition that $f-y^{\prime} f_{y^{\prime}}$ is continuous will in general not be satisfied. Moreover, the Weierstrass condition will in general not be satisfied by all the solutions of the family. So far as I can see, to obtain an effective extension of the Jacobi condition to discontinuous solutions, it is necessary to proceed step by step past one corner at a time.

Tonelli has obtained stronger results than mine in one respect, namely in showing that the Weierstrass and Legendre conditions hold almost everywhere, and without the assumption that $y^{\prime}$ is bounded. $\ddagger$ For the parametric problem Tonelli assumes only that the minimizing curve is rectifiable and then

$*(14)$.

$\dagger(16)$.

$\ddagger$ See (56), vol. II, pp. 83, 317, 511, 557. 
obtains the du Bois-Reymond equations almost everywhere, a stronger result than Hahn's in some respects.*

3. Corners related to Restrictions on the Position or Direction of Admissible Curves. Let us turn now to some types of problems in which discontinuities in the direction of the minimizing curve are connected with restrictions on the position or direction of admissible curves. In 1831 Goldschmidt $\dagger$ discovered the very interesting and well known example in which the curve generating a surface of revolution of minimum area is composed of a piece of the axis of revolution together with two line segments perpendicular to it. In this example the boundary restriction is $y \geqq 0$.

In 1879 Weierstrass $\ddagger$ gave the inequality $T \geqq 0(\leqq 0)$ which replaces the Euler equation $T=0$ for a piece of the minimizing curve lying along the boundary of the region of admissible curves. Todhunter gave this for the non-parametric form of the problem in 1871.§ Weierstrass $\$$ also obtained the condition holding at the point where the minimizing curve meets the boundary, namely, $\mathcal{E}\left(x, y, x^{\prime}, y^{\prime}, \bar{x}^{\prime}, \bar{y}^{\prime}\right)=0$, where $x^{\prime}: y^{\prime}$ gives the direction of the extremal arc interior to the region, and $\bar{x}^{\prime}: \bar{y}^{\prime}$ gives the direction of the boundary curve. If the problem is regular on the boundary, this condition implies tangency, and no corners are present. For this case Bliss derived sufficient conditions for a minimum in 1904. It would be desirable to carry through the theory without the hypothesis of regularity.

Problems in three dimensions of the foregoing type were treated in 1914 by Bliss and Underhill.** In 1878 Mayer stated the law of the conservation of the isoperimetric constant or multiplier. $\dagger^{\dagger}$ This law says that the multiplier is the same for all portions of the minimizing curve interior to the region.

* See (56), vol. II, pp. 89, 318, 486, 557.

$\dagger$ (25). See also MacNeish (26) and Sinclair (27).

$\ddagger$ See Bolza (54), p. 395. I was not able to find this in Weierstrass' Werke nor in a copy of notes on his 1879 lectures.

$\S(48)$, p. 13.

I (51), vol. 7, p. 307 .

|| See Bolza (54), pp. 400-407, Lindeberg (19).

** (22). See Hadamard (55), p. 179. Also an example of a singular case in Todhunter (48), p. 128.

$\dagger \dagger(17)$. 
It was proved by Weierstrass in $1879 .^{*}$ Hadamard indicated the conditions along the boundary for isoperimetric problems in $1907 . \dagger$

The case when one point of the minimizing curve is required to be on the boundary of the region of admissible curves was considered by Weierstrass. $\ddagger$ The condition holding at this point is

$$
\frac{|\sin \delta|}{|\sin \bar{\delta}|}=\frac{\mathcal{E}(x, y, \cos \bar{\theta}, \sin \bar{\theta}, \cos \theta, \sin \theta)}{\mathcal{E}(x, y, \cos \theta, \sin \theta, \cos \bar{\theta}, \sin \bar{\theta})},
$$

where $\theta$ and $\bar{\theta}$ are the slope angles of the two arcs of extremals meeting at the point, and $\delta$ and $\bar{\delta}$ are the angles at which they meet the boundary. This is an extension of the law of reflection of light. No further work seems to have been done for this problem, but Bliss and Mason state that their results for the problem with discontinuous integrand $\S$ can be interpreted for this problem, and hence we have a sufficiency theorem as well as necessary conditions.

When there are restrictions on the slope or curvature of admissible curves, the problem becomes more complicated. Todhunter has many examples in his Researches. $\uparrow$ Zermelo gave a very brief and sketchy account of the situation in 1902.\| In the same year Kneser gave a definitive treatment of Newton's problem of the solid of revolution of minimum resistance, with restrictions on the slopes of admissible curves.** Hadamard $\dagger \dagger$ considered the problem of Mayer with some inequalities among the side conditions, and obtained inequalities for the multipliers occurring in the multiplier rule. Finally in 1914 Bolza $\ddagger \ddagger$ undertook to study his generalization of the problems of Mayer and Lagrange, with inequalities replacing equalities in some of the differential equations, finite equations, and

* (51), vol. 7, p. 248.

$\dagger(20)$.

$\ddagger(51)$, vol. 7, p. 311. See an example in Todhunter (48), p. 128.

$\S(33)$.

T (48), pp. 38, 73, 103, 109, 111, 142, 143, 170, 201, 252.

$\|(23)$.

** (31). See Bolza (54) pp. 407-418. See Lecat (32) for a bibliography of Newton's problem.

$\dagger \dagger(55)$, p. 248.

$\ddagger \ddagger(24)$. 
end conditions. Neither Hadamard nor Bolza considers the conditions which must hold at a point of transition where an inequality holding in the strict sense along the minimizing curve becomes an equality. It should be noted also that the assumption of normality for the minimizing arc excludes the special case of the plane problem with boundary restrictions, as well as other simple cases. There is need in this domain for a further study of special cases, in order that a general theory may be properly oriented.

4. Problems in which the Integrand is Discontinuous. A minimizing problem in which the integrand is discontinuous arises in the consideration of the path of light through a medium whose refractive power changes abruptly. The general problem of this type in the plane was treated by Bliss and Mason in 1906.* The corner condition at the curve of discontinuity is that $F_{x^{\prime}} d x+F_{y^{\prime}} d y$ shall be continuous across this curve, where $d x: d y$ gives the direction of the curve of discontinuity. This condition is due to Hilbert (Lectures 1904/5). $\dagger$ Bliss and Mason obtained the extension of the Jacobi condition by means of the envelope theorem, and discussed sufficient conditions. Their work is apparently easily extensible to any number of dimensions and any number of discontinuities. $\ddagger$ It would be of interest to consider the second variation for this problem.

More recently Roos $\S$ has discussed a generalization of Bolza's general problem, in which the integrand may be discontinuous. Roos formulates the problem as follows. The expression to be minimized is the sum of two integrals

$$
\begin{aligned}
I & =\int_{x_{1}}^{\xi} g\left(x, y, y^{\prime}, x_{1}, y_{1}, x_{2}, y_{2}, \xi, y(\xi)\right) d x \\
& +\int_{\xi}^{x_{2}} h\left(x, y, y^{\prime}, x_{1}, y_{1}, x_{2}, y_{2}, \xi, y(\xi)\right) d x
\end{aligned}
$$

whose integrands may depend both on the given end values $\left(x_{1}, y_{1}\right),\left(x_{2}, y_{2}\right)$, and on the coordinates of the transition point $(\xi, y(\xi))$. Admissible curves satisfy the $m$ differential equations

*(33).

$\dagger$ See Bolza (54), p. 390, footnote (2).

$\ddagger$ See Miles (34), (35).

$\S(36)$. 
$\phi_{\alpha}\left(x, y, y^{\prime}\right)=0$ and the end-point conditions have the form $\theta_{\mu}\left(x_{1}, y_{1}, x_{2}, y_{2}\right)=0$. There is no restriction placed on the point of transition or discontinuity, so that Roos' work does not include the special case of Bliss and Mason. Roos obtains the conditions associated with the first variation, namely, the extension of the Lagrange equations, the corner conditions, and the transversality conditions.

A special case of Roos' problem which has been studied more completely is the problem of minimizing the sum of an integral and a point function,

$$
J=\int f\left(x, y, y^{\prime}\right) d x+\phi(\xi, \eta)
$$

where $\eta=y(\xi)$. Hadamard* gave the corner conditions which must hold at the point $(\xi, y(\xi))$, namely,

$$
\left(f-y^{\prime} f_{y^{\prime}}\right)^{+}-\left(f-y^{\prime} f_{y^{\prime}}\right)^{-}=\partial \phi / \partial \xi, f_{y^{\prime}}^{+}-f_{y^{\prime}}^{-}=\partial \phi / \partial \eta .
$$

Miss Sinclair studied an example in 1909. $\dagger$ In 1922 Clarke made a study of necessary conditions and sufficient conditions in the plane problem. Besides the corner conditions already mentioned, he obtained an inequality $T \geqq 0$ which must hold at the corner $(\xi, \eta)$. Then he obtained an extension of the Jacobi condition by means of an envelope theorem. I have a strong feeling that Clarke did not unravel the full significance of his condition $T \geqq 0$.

5. Multiple Integrals. I shall speak very briefly of discontinuous solutions of multiple integral problems, because but little has been done. Kobb obtained the edge conditions for the double integral in parametric form in 1892.\$ These are that

$$
\frac{\partial F}{\partial x_{u}} d v-\frac{\partial F}{\partial x_{v}} d u
$$

and two similar expressions in which $x$ is replaced by $y$ and $z$ respectively, shall all be continuous across a curve of discontinuity of the derivatives $x_{u}, x_{v}, y_{u}, y_{v}, z_{u}, z_{v}$, where $d u: d v$

* (55), §159.

$\dagger(38)$.

$\ddagger(37)$.

$\S(39 a)$, p. 84. For the isoperimetric problem, (39b), p. 325. 
determines the direction of the curve of discontinuity. Kobb took up also the case of one-sided variations and the case when the minimizing surface is required to have a curve in common with the boundary.* The former problem was treated more completely by Fischer, $\dagger$ who gave sufficient conditions for a minimum with respect to one-sided variations. Kobb's edge conditions (2) can be easily obtained from various fundamental lemmas for double integrals $\$$ which lead to forms of the Lagrange equation analogous to the du Bois-Reymond form for simple integrals.

In 1909 Haar and von Kármán§ considered triple integrals with differential inequalities as side conditions, but they obtained no satisfactory conclusions. This is not surprising, since the Lagrange multiplier rule for the problem of minimizing a double integral with differential equations as side conditions seems never to have been proved in satisfying fashion.

6. Minimizing Curves that are Actually Discontinuous. Finally I wish to speak of a type of calculus of variations problem proposed by Razmadzé T in which certain curves with actual discontinuities are admitted to consideration as paths of integration. Razmadzé seems to have been motivated largely by the example given by Weierstrass $\|$ of an integral which has a finite lower bound but no minimum in the class of continuous curves. This example is

$$
J=\int x^{2} y^{\prime 2} d x
$$

where the curve is to join two points lying on opposite sides of the $y$-axis and having different ordinates. The integral has the value zero along the discontinuous curve composed of two segments parallel to the $x$-axis. This curve is approached by a sequence of continuous curves $\left\{C_{n}\right\}$ such that $\lim J\left(C_{n}\right)=0$. This property leads to Razmadzé's definition of admissible discontinuous curves for the problem of minimizing the integral

* (39b), p. 337-343.

$\dagger(43)$.

$\ddagger$ See Mason (40), Haar (41), Schauder (42).

$\S(44)$.

I (46). Perhaps earlier in (45).

II See (51), vol. 2, p. 53. 
$J=\int f\left(x, y, y^{\prime}\right) d x$. A curve $D, y=y(x)$, having a single ordinary discontinuity at $x=x_{0}$ is admissible if a sequence $\left\{C_{n}\right\}$ of continuous admissible curves exists such that $\lim C_{n}=D$, $\lim J\left(C_{n}\right)=J(D)$. This condition on admissible discontinuous curves may limit the abscissa of the discontinuity to a fixed value $x_{0}$, as in the Weierstrass example. This is case I. Case II is that in which the abscissa of the point of discontinuity is freely variable. Razmadzé implicitly requires also that the ordinates be freely variable. In case I no new theory is required, so far as discontinuous comparison curves are concerned. In case II Razmadzé obtains a discussion of the conjugate point conditions strikingly analogous to that of Carathéodory, Bolza, and Dresden for the case of minimizing arcs with corners. He also discusses sufficient conditions for a strong minimum in the field $F_{0}$ of discontinuous comparison curves. He speaks of two fields of continuous comparison curves. The field $F_{1}$ is composed of sequences of continuous curves approaching discontinuous comparison curves, and the field $F_{2}$ is composed of sequences of continuous curves approaching the discontinuous minimizing curve. In both cases the value of the integral must approach the value along the discontinuous limiting curve. It is apparent that the fields $F_{1}$ and $F_{2}$ are rather ill-defined ideas. Now all that Razmadzé pretends to prove is that certain conditions are sufficient for a discontinuous curve $D$ to give a smaller value to $J$ than do the curves of a given sequence of $F_{1}$ or $F_{2}$, after a sufficiently large number of terms have been discarded. Nevertheless in his summary occurs the surprising statement that if the discontinuous curve $D$ minimizes $J$ in the field $F_{0}$ of discontinuous comparison curves, then $D$ gives a smaller value to $J$ than every curve of the field $F_{1}$, and (provided a supplementary condition is satisfied) than every curve of the field $F_{2}$. There are also minor objections to Razmadzé's paper which contribute to the impression that it has little value.

However, Sakellariou* was sufficiently interested to attempt a parallel discussion for the problem in parametric form. The points of rupture are still restricted to have the same abscissa. Sakellariou does not attempt a thoroughgoing investigation as

* (47). 
does Razmadzé, but a more sweeping objection to his paper can be made, since his first theorem, fundamental to all that follows, is quite obviously in error.

\section{BIBLIOGRAPHY}

\section{A. The Minimizing Curve is Interior to the Region} of Admissible Curves

1. Erdmann, Über unstetige Lösungen in der Variationsrechnung, Journal für Mathematik, vol. 82 (1876), pp. 21-30.

2. Carathéodory, Über die diskontinuierlichen Lösungen in der Variationsrechnung, Dissertation, Göttingen, 1904, (v+71 pp.).

3. Carathéodory, Über die starken Maxima und Minima bei einfachen Integralen, Mathematische Annalen, vol. 62 (1906), pp. 449-503.

4. Bolza, The determination of the conjugate points for discontinuous solutions in the calculus of variations, American Journal of Mathematics, vol. 30 (1908), pp. 209-21.

5. Dresden, The second derivatives of the extremal integral, Transactions of this Society, vol. 9 (1908), p. 467.

6. Carathéodory, Sur les points singuliers du problème du calcul des variations dans le plan, Annali di Matematica, vol. 21 (1913), p. 153.

7. Razmadzé, Sur une condition de minimum nécessaire pour les solutions anguleuses dans le calcul des variations, Bulletin de la Société Mathématique de France, vol. 51 (1923), p. 223.

8. Graves, Discontinuous solutions in space problems of the calculus of variations, American Journal of Mathematics, vol. 52 (1930), pp. 1-28.

9. Hefner, The condition of Mayer for discontinuous solutions of the Lagrange problem, Dissertation, Chicago.

10. Rider, $A$ note on discontinuous solutions in the calculus of variations, this Bulletin, vol. 23 (1917), pp. 237-240.

11. Sakellariou, (a) Über Variationsrechnung im Raume, Tôhoku Mathematical Journal, vol. 13 (1918), pp. 15-24. (b) The space problem of the calculus of variations, Rendiconti di Palermo, vol. 44 (1920), pp. 53-68.

12. Sakellariou, Sur les solutions discontinus du problème $d u$ calcul des variations dans l'espace à $n$ dimensions, (a) Congrès International des Mathématiciens, Strasbourg, 1920, pp. 351-355, (b) Bulletin de la Société Mathématique de Grèce, vol. 3 (1922), pp. 37-46.

\section{Infinitely many Discontinuities in the DiRection of the Minimizing Curve}

13. Whittemore, Lagrange's equation in the calculus of variations, and the extension of a theorem of Erdmann, Annals of Mathematics, (2), vol. 2 (1901), pp. 130-6.

14. Hahn, Über die Herleitung der Differentialgleichungen der Variationsrechnung, Mathematische Annalen, vol. 63 (1907), pp. 253-72.

15. Fubini, Sulla definizione di arco di una curva e dell'integrale di Weierstrass, che si presenta nel calcolo delle variazioni, Rendiconti dei Lincei, (5), vol. 24 (1915), pp. 127-129. 
16. Graves, The derivative as independent function in the calculus of variations, Dissertation, Chicago, 1924.

\section{B. Corners related to Restrictions on Position or Direction of Admissible Curves}

17. Mayer, Die Kriterien des Maximums und Minimums der einfachen Integrale in den isoperimetrischen Problemen, Mathematische Annalen, vol. 13 (1878), p. 65, footnote.

18. Bliss, Sufficient conditions for a minimum with respect to one-sided variations, Annals of Mathematics, (2), vol. 5 (1904), pp. 477-92.

19. Lindeberg, Über ein Problem der Variationsrechnung, Översigt af Finska Vetenskaps-Societetens Förhandlingar, vol. 51 (1908-09), Afd. A, No. 21.

20. Hadamard, Sur quelques questions de calcul des variations, Annales de l'École Normale, (3), vol. 24 (1907), p. 203.

21. Frank, Unstetige Lösungen beim Prinzip der kleinsten Wirkung, Monatshefte für Mathematik und Physik, vol. 20 (1909), pp. 189-92.

22. Bliss and Underhill, The minimum of a definite integral for unilateral variations in space, Transactions of this Society, vol. 15 (1914), p. 291.

23. Zermelo, Zur Theorie der kürzesten Linien, Jahresbericht der Deutschen Mathematiker Vereinigung, vol. 11 (1902), pp. 184-187.

24. Bolza, Über Variationsprobleme mit Ungleichungen als Nebenbedingungen, Mathematische Abhandlungen H.A.Schwarz, gewidmet, Berlin, 1914, p. 1.

\section{The Surface of Revolution of Minimum Area}

25. Goldschmidt, Determinatio superficiei minimae rotatione curvae data duo puncta jungentis circa datum axem ortae, Göttingen Prize Essay (1831).

26. MacNeish, Concerning the discontinuous solution in the problem of the minimum surface of revolution, Annals of Mathematics, (2), vol. 7 (1906), pp. 72-80.

27. Sinclair, The absolute minimum in the problem of the surface of revolution of minimum area, Annals of Mathematics, (2), vol. 9 (1907-08), pp. 151-5.

\section{Newton's Problem}

28. Starkoff, Sur la surface offrant la moindre résistance au mouvement dans un fluide incompressible, Société des Naturalistes de la nouvelle Russie (Odessa), Mémoires de la section mathématique, vol. 5 (1884), pp. 49-136, and vol. 6 (1885), pp. 47-56.

29. August, Über die Rotationsflache kleinsten Widerstandes, Journal für Mathematik, vol. 103 (1888), p. 1.

30. Armanini, Sulla superficie de minima resistenza, Annali di Matematica, (3), vol. 4 (1900), p. 131-150.

31. Kneser, Ein Beitrag zur Frage nach der zweckmässigsten Gestalt der Geschossspitzen, Archiv der Mathematik und Physik, (3), vol. 2 (1902), pp. 267-278.

32. Lecat, Bibliographie du problème de Newton, Intermédiaire der Mathématiciens, (1), vol. 23 (1916), pp. 81-4. 


\section{Discontinuous Integrands}

33. Bliss and Mason, A problem in the calculus of variations in which the integrand is discontinuous, Transactions of this Society, vol. 7 (1906), pp. 325-36.

34. Miles, Note on the isoperimetric problem with discontinuous integrand (Abstract only), this Bulletin, vol. 18 (1912), p. 333.

35. Miles, Some properties of space curves minimizing a definite integral with discontinuous integrand, this Bulletin, vol. 20 (1913), pp. 11-19.

36. Roos, A general problem of minimizing an integral with discontinuous integrand, Transactions of this Society, vol. 31 (1929), pp. 58-70.

37. Clarke, On the minimum of the sum of a definite integral and a function of a point, Dissertation, Chicago, 1922.

38. Sinclair, Concerning a compound discontinuous solution in the problem of the surface of revolution of minimum area, Annals of Mathematics, (2), vol. 10 (1909), pp. 55-80.

\section{Multiple Integrals}

39. Kobb, Sur les maxima et les minima des intégrales doubles, Acta Mathematica, (a) vol. 16 (1892), p. 65, (b) vol. 17 (1893), p. 321.

40. Mason, Beweis eines Lemmas der Variationsrechnung, Mathematische Annalen, vol. 61 (1905), pp. 450-2.

41. Haar, Über die Variation der Doppelintegrale, Journal für Mathematik, vol. 149 (1919), p. 1.

42. Schauder, Über die Umkehrung eines Satzes aus der Variationsrechnung, Acta Litterarum ac Scientiarum, Szeged, vol. 4 (1928), pp. 38-50.

43. Fischer, Minima of double integrals with respect to one-sided variations, Annals of Mathematics, (2), vol. 16 (1915), pp. 162-8.

44. Haar und von Kármán, Zur Theorie der Spannungszustände in plastischen und sandartigen Medien, Göttinger Nachrichten, 1909, pp. 204-218.

\section{E. Minimizing Curves Actually Discontinuous}

45. Razmadzé, Über unstetige Lösungen mit einem Unstetigkeitspunkt in der Variationsrechnung, Bulletin de l'Université Tiflis, vol. 2 (1922-23), pp. 282-312. (Contents unknown.)

46. Razmadzé, Sur les solutions discontinues dans le calcul des variations, (a) Mathematische Annalen, vol. 94 (1925), pp. 1-52. (b) International Mathematical Congress, Toronto, 1924, vol. 1, pp. 561-588.

47. Sakellariou, Über die nicht-stetigen Lösungen in der Variationsrechnung, Rendiconti di Palermo, vol. 51 (1927), pp. 38-48.

\section{F. Books}

48. Todhunter, Researches in the Calculus of Variations, London, 1871.

49. Carll, Treatise on the Calculus of Variations, New York, 1881.

50. Pascal, Varintionsrechnung, Leipzig, 1899, §30.

51. Weierstrass, Werke.

52. Hancock, Lectures on the Calculus of Variations.

53. Kneser, Lehrbuch der Variationsrechnung, Braunschweig, 1900. 
54. Bolza, Vorlesungen über Variationsrechnung, Chap. VIII.

55. Hadamard, Lȩ̧ons sur le Calcul des Variations.

56. Tonelli, Fondamenti di Calcolo delle Variazioni, vols. I, II. Bologna, $1921,1923$.

\section{G. Miscellaneous Early Papers}

57. Challis, On the brachystochronous course of a ship, London and Edinburgh Philosophical Magazine, (3), vol. 4 (1834), p. 33.

58. Airy, On a supposed failure of the calculus of variations, London, Edinburgh and Dublin Philosophical Magazine, (4), vol. 22 (1861), p. 12.

59. Challis, London, Edinburgh and Dublin Philosophical Magazine, (a), On a problem in the calculus of variations, (4), vol. 32 (1866), pp. 278-83. (b), On integrating differential equations by factors and differentiation, with application in the calculus of variations, vol. 46 (1873), pp. 388-98; (c), Solution of a problem in the calculus of variations, vol. 22 (1861), pp. 108-11; (d), On the principle of discontinuity in solutions of problems of the calculus of variations, vol. 24 (1862), pp. 196-201; (e), The solution of a problem in the calculus of variations by a nere method, vol. 31 (1866), p. 218-27; (f), On an extension of the principles of the calculus of variations, vol. 32 (1866), pp. 45-54.

60. Todhunter, London, Edinburgh and Dublin Philosophical Magazine, (a), On a problem in the calculus of variations, vol. 31 (1866), pp. 425-7; (b), On a problem in the calculus of variations, vol. 32 (1866), pp. 199-205.

61. Wilkinson, On false discontinuity, with illustrations from Fourier's theorem and the calculus of variations, Cambridge, 1871, $10 \mathrm{pp}$.

62. Wilkinson, Review of Todhunter's "Researches," Messenger of Mathematics, vol. 3 (1874), p. 184.

63. Maxwell, On a problem in the calculus of variations in which the solution is discontinuous, Proceedings of the Cambridge Philosophical Society, vol. 2 (1876), pp. 294-5 (=Scientific papers, vol. 2, p. 310).

64. Starkoff, Sur la résolution des problèmes géométriques par le calcul des variations, Bulletin de la Société Mathématique de France, vol. 13 (1885), pp. 132-42.

65. Ssónin, Sur un problème du calcul des variations, Société des Naturalistes de la Nouvelle Russie (Odessa), Mémoires de la Section Mathématique, vol. 6 (1885), pp. 1, 93.

66. Culverwell, Researches in the calculus of variations, Part VI, The theory of discontinuous or compounded solutions, Proceedings of the London Mathematical Society, vol. 26 (1894-5), pp. 345-64.

67. Zimmerman, Discontinuous solutions in the calculus of variations, Mémoires de l'Université Odessa, (1896), pp. 165-462.

68. Sabinine, On discontinuous solutions in the calculus of variations, Mathematical Series of the Moscow Mathematical Society, vol. 20 (1898), pp. 255-92.

The University of Chicago 